\section{ALIMENTAÇÃO E DIABETES MELLITUS: PERCEPÇÃO E CONSUMO ALIMENTAR DE IDOSOS NO INTERIOR DE PERNAMBUCO}

Feeding and Diabetes Mellitus: perception and food
consumption by elderlypeoplein thehinterlandsofPernambuco

Alimentación y Diabetes Mellitus: percepción y consumo de alimentos de mayores del interior de Pernambuco

\section{RESUMO}

Objetivo: Avaliar o conhecimento sobre alimentação relacionada ao diabetes e identificar fatores que possam interferir na adesão à terapia nutricional e nas escolhas alimentares de participantes de um Centro de Convivência de Idosos em Sairé-PE. Métodos: Estudo quantitativo, transversal e descritivo com 39 frequentadores do referido centro, de julho a agosto de 2014, portadores ou não de diabetes mellitus. Aplicaram-se dois questionários para avaliar dados socioeconômicos, conhecimento alimentar e fatores culturais, e verificar o consumo de alimentos de alto e baixo índice glicêmico. Os dados foram analisados através do Programa Assistat versão 7.0 beta. Resultados: Observou-se que a maioria possui conhecimentos sobre tipos de alimentos que podem influenciar no tratamento de diabetes mellitus, pois $51,2 \%(\mathrm{n}=20)$ disseram conhecer algum alimento que pode reduzir os riscos de acometimento ou auxiliar no tratamento do diabetes. A maioria disse ter adquirido esse conhecimento por meio de televisão $(35 \% ; n=7)$ e conversa com conhecidos $(35 \% ; n=7)$. Verificando o consumo alimentar, constatou-se maior consumo de alimentos com alto índice glicêmico. Porém, entre os diabéticos, viu-se que os alimentos de baixo índice glicêmico são consumidos mais vezes por semana. Conclusão: Considerou-se adequado o nível de conhecimento em relação à alimentação e diabetes, mas viu-se que fatores socioeconômicos e culturais podem interferir na adesão à dietoterapia ou nas escolhas alimentares dos indivíduos. Contudo, o consumo alimentar se mostrou mais adequado entre os diabéticos.

Descritores: Consumo Alimentar; Diabetes Mellitus; Idoso.

\section{ABSTRACT}

Objective: To evaluate the knowledge of diabetes diet and identify factors that may interfere with the adherence to nutritional therapy and food choices of participants in a Community Center for the Elderly in Sairé, PE. Methods: A quantitative, descriptive and cross-sectional study, which evaluated 39 attendees of that center, from July to August 2014, with or without diabetes mellitus. Two questionnaires were applied to assess socioeconomic data, nutrition knowledge and cultural factors, and check the consumption of food with high and low glycemic index. Data was analyzed using the Assistat Program version 7.0 beta. Results: The majority of the respondents have knowledge about types of foods that may influence the treatment of diabetes mellitus, as $51.2 \%(n=20)$ reported knowing some food that can reduce the risk for diabetes onset or assist in its treatment. Most of the participants reported having acquired such knowledge through the television $35 \%(n=7)$ and conversation with peers $35 \%(n=7)$. Evaluation of the food intake evidenced higher consumption of foods with high glycemic index. However, among diabetic patients, foods with low glycemic index are consumed more times per week. Conclusion: The knowledge about nutrition and diabetes mellitus was considered adequate, but socioeconomic and cultural factors may interfere in the adherence to diet therapy for diabetes or in the food choices made by the individuals. However, food consumption was considered appropriate among diabetics.

Descriptors: Food Consumption; Diabetes Mellitus; Aged.
Artigo Original

Barbosa $^{(1)}$

Ana Maria Rampeloti Almeida ${ }^{(1)}$

Mariana Andrade Figueiredo(1)

Adriana Guimarães

Negromonte $^{(1)}$

Jordana Sirlaide Lima da Silva ${ }^{(1)}$

Marcia Gabrielle Silva Viana ${ }^{(1)}$

Georgia Karoline Cavalcante

Galvão $^{(1)}$

1) Centro Universitário do Vale do Ipojuca UNIFAVIP/DeVry - Caruaru (PE) - Brasil

Recebido em: 25/08/2015 Revisado em: 16/09/2015 Aceito em: 21/09/2015 


\section{RESUMEN}

Objetivo: Evaluar el conocimiento sobre la alimentación relacionada a la diabetes e identificar los factores que puedan contribuir para la adhesión de la terapia nutricional y las elecciones alimentarias de los participantes de un Centro de Convivencia para Mayores de Sairé-PE. Métodos: Estudio cuantitativo, transversal y descriptivo con 39 mayores del referido centro entre Julio y Agosto de 2014, portadores o no de diabetes mellitus. Se aplicó dos cuestionarios para evaluar los datos socioeconómicos, el conocimiento alimentario y factores culturales y verificar el consumo de alimentos de bajo y alto índice glicémico. Los datos fueron analizados a través del Programa Assistat versión 7.0 beta. Resultados: Se observó que la mayoría tiene conocimientos de los tipos de alimentos que pueden influir en el tratamiento de la diabetes mellitus pues el 51,2\% (n=20) afirmaron conocer algún alimento que puede reducir los riesgos de tener la diabetes o auxiliar en su tratamiento. La mayoría afirmó haber adquirido este conocimiento a través de la televisión $(35 \% ; n=7)$ y charla con conocidos $(35 \% ; n=7)$. Al verificar el consumo de alimentos, se constató mayor consumo de alimentos con alto indice glicémico. Sin embargo, entre los diabéticos, los alimentos de bajo índice glicémico son consumidos más veces a la semana. Conclusión: Se consideró el nivel de conocimiento sobre la alimentación y la diabetes adecuado pero se observó que factores socioeconómicos y culturales pueden influir en la adhesión de la dietoterapia o en la elección de los alimentos de los individuos. Sin embargo, el consumo de alimentos se mostró más adecuado entre los diabéticos.

Descriptores: Consumo de Alimentos; Diabetes Mellitus; Anciano.

\section{INTRODUÇÃO}

O diabetes mellitus é um grupo de doenças metabólicas caracterizadas por um quadro de hiperglicemia resultante de defeitos na ação, na secreção de insulina ou em ambos os casos. Quando o indivíduo é acometido pela doença por um longo período de tempo, tem-se como decorrência a redução da atividade de vários órgãos, como rins, olhos, coração, vasos sanguíneos e nervos ${ }^{(1)}$.

A incidência de diabetes mellitus no mundo cresce de forma alarmante. Em 1985, o número de adultos diabéticos, a nível mundial, chegava a 30 milhões, avançando no ano de 1995 para 135 milhões. Em 2002, já alcançava 173 milhões de pessoas, e a previsão para 2030 é que esses números possam chegar à casa dos 300 milhões $^{(2)}$.

No Brasil, ao final da década de 1980, relatava-se que $8,0 \%$ da população entre 30 e 69 anos, residente em regiões metropolitanas, era diabética. Em 2005, estimava-se que $11,0 \%$ dos que tinham 40 anos ou mais eram acometidos pela doença, o que correspondia a cerca de cinco milhões e meio de pessoas, de acordo com a população brasileira estimada pelo IBGE no respectivo ano $^{(3)}$.
Muitas pessoas, quando recebem o diagnóstico do diabetes mellitus tipo 2, já apresentam as complicações crônicas da doença, o que interfere em sua qualidade de vida e proporciona maiores gastos ao serviço de saúde ${ }^{(4)}$, os quais variam de acordo com a prevalência local e a complexidade do tratamento disponível. Essas complicações também podem levar à morte prematura, perda de produtividade no local de trabalho e aposentadoria precoce, representando uma carga a mais para a sociedade, além de menor qualidade de vida não apenas para o indivíduo, mas também para sua família ${ }^{(3)}$.

No caso do diabetes, sabe-se das dificuldades encontradas pelos indivíduos para aderir ao tratamento da doença, e da influência de fatores como baixo nível de escolaridade, crenças, não aceitação da doença, entre outros, que podem impedir o indivíduo de adotar mudanças necessárias para cuidar de sua saúde ${ }^{(5)}$. Há possibilidade de que os níveis de informação recebida pela população sobre os fatores de risco para desenvolvimento de doenças crônicas não transmissíveis estejam relacionados a diferenças regionais, socioeconômicas e culturais ${ }^{(6)}$.

Considerando a importância da alimentação e da adesão à terapia nutricional para a melhoria da qualidade de vida da população e o sucesso do tratamento do diabetes mellitus, torna-se importante estudar o conhecimento sobre alimentação no diabetes, visando contribuir com a literatura e proporcionando dados para a reflexão e realização de novas pesquisas e estratégias na área de nutrição social que foquem a prevenção de doenças e agravos.

Diante do exposto, o objetivo deste estudo foi avaliar o conhecimento sobre alimentação relacionada ao diabetes mellitus, identificar fatores que possam interferir na adesão à terapia nutricional e nas escolhas alimentares, e observar o consumo alimentar real dos participantes de um Centro de Convivência de Idosos no município de Sairé-PE.

\section{MÉTODOS}

Estudo quantitativo, de natureza transversal e descritiva, realizado com frequentadores do Centro de Convivência de Idosos (CCI) na cidade de Sairé-PE, entre os meses de julho e agosto de 2014. Compuseram a amostra 39 pessoas, representando $65 \%$ do total dos indivíduos cadastrados no CCI. Participaram da pesquisa indivíduos de ambos os sexos, portadores ou não de diabetes mellitus, que estavam devidamente cadastrados e participavam regularmente das atividades oferecidas pelo centro de convivência, estando presentes nos dias da coleta de dados.

Realizou-se a coleta de dados por intermédio da aplicação de questionário adaptado ${ }^{(7)}$, incluindo questões socioeconômicas (sexo, idade, estado civil, escolaridade, renda mensal no domicílio, número de componentes na 
família e se o indivíduo é acometido pelo diabetes ou não). Além de traçar o perfil da população avaliada, o questionário abordou perguntas com o intuito de avaliar os conhecimentos sobre alimentos que podem aumentar o risco de desenvolver ou agravar o quadro de diabetes mellitus, contendo nove frases relacionadas à alimentação e ao diabetes, às quais os entrevistados deveriam responder "verdadeiro" ou "falso" para cada uma.

A identificação dos fatores culturais que poderiam interferir na adesão à terapia nutricional e nas escolhas alimentares dos participantes foi averiguada através de cinco questões inseridas no questionário: se o indivíduo tem conhecimento de algum alimento/preparação que pode reduzir os riscos ou auxiliar no tratamento do diabetes; um exemplo do alimento; se o indivíduo já fez uso deste; onde ouviu indicações ou quem the falou sobre o alimento; se já foi a uma consulta com algum profissional de saúde para receber orientação sobre alimentação saudável e diabetes; e se já foi a algum nutricionista.

As práticas alimentares reais foram avaliadas por meio de um questionário de frequência de consumo alimentar (QFCA), aplicado em dia diferente da entrevista sobre conhecimento alimentar, no qual se agrupou os alimentos em dois grupos: alimentos de alto índice glicêmico (macaxeira, bolacha água e sal, farofa, melancia, melão, pão francês e torradas) e alimentos de baixo índice glicêmico (banana, laranja, arroz integral, leite desnatado, leite integral, feijão, iogurte, iogurte diet e refrigerante diet). A determinação, bem como a classificação do índice glicêmicos dos alimentos, em alto e baixo, foi feita de acordo com estudos prévios $^{(8-10)}$. A frequência de consumo foi avaliada de acordo com o consumo semanal, sendo considerado o consumo inferior a três vezes, de três a cinco vezes e diariamente.
A análise estatística foi feita no Programa Assistat versão 7.0 beta. Realizou-se uma análise de variância para avaliar se os valores encontrados para ingestão de alimentos com baixo e alto índice glicêmico apresentavam diferença estatisticamente significativa. A pesquisa obedeceu à Resolução 466/12 do Conselho Nacional de Saúde e foi aprovada em 05 de junho de 2014 pelo Comitê de Ética em Pesquisa do Centro Universitário do Vale do Ipojuca, sob protocolo ${ }^{\circ}$ 0010/2014. Todos os participantes deste estudo assinaram o Termo de Consentimento Livre Esclarecido (TCLE).

\section{RESULTADOS}

A pesquisa identificou que 39 idosos frequentavam semanalmente o Centro de Convivência de Idosos, e a maioria era mulher $(92,3 \%)$. A média de idade desses indivíduos ficou em 67,2 anos, sendo o mais jovem de 48 anos e o mais idoso de 84 anos. De acordo com o perfil socioeconômico, observou-se que 43,5\% ( $\mathrm{n}=17)$ deles eram casados e $33,3 \%(n=13)$, viúvos.

No tocante à escolaridade, observou-se que uma parcela significativa era analfabeta $(23,0 \% ; n=9)$, a maioria $(74,3 \%$; $\mathrm{n}=29$ ) não tinha concluído o ensino fundamental e apenas um deles havia concluído o ensino médio. Com relação à renda mensal, percebeu-se que um pouco mais da metade dos entrevistados $(56,4 \% ; \mathrm{n}=22)$ relatou receber entre 1 e 2 salários mínimos, embora $28,2 \%(\mathrm{n}=11)$ informaram dispor de 1 ou menos que 1 salário mínimo ao mês. A maior parte dos entrevistados $(30,7 \%)$ residia com 1 e 2 pessoas $(30,7 \%), 8$ pessoas informaram morar sozinhas $(20,5 \%)$ e apenas 2 referiram residir com 5 pessoas.

Dos 39 indivíduos, encontramos 35,9\% ( $\mathrm{n}=14)$ com diabetes diagnosticada e um deles não sabia informar se era

Tabela I - Conhecimento alimentar relacionado ao diabetes mellitus, de frequentadores de um Centro de Convivência de Idosos na cidade de Sairé. Pernambuco, 2014.

\begin{tabular}{lcccc}
\hline \multirow{2}{*}{ Questões } & \multicolumn{2}{c}{ Resposta correta } & \multicolumn{2}{c}{ Resposta errada } \\
\cline { 2 - 5 } & $\mathbf{n}$ & $\mathbf{\%}$ & $\mathbf{n}$ & $\mathbf{\%}$ \\
\hline Pão, bolacha e torrada interferem no açúcar no sangue & 27 & 69,2 & 12 & 30,7 \\
O diabético não pode comer melão e melancia à vontade & 30 & 76,9 & 9 & 23,0 \\
O diabético pode comer laranja e banana livremente & 37 & 94,8 & 2 & 5,1 \\
O diabético não pode comer leite e iogurte livremente & 34 & 87,1 & 5 & 12,8 \\
Fontes de fibras ajudam a controlar o açúcar e o colesterol & 35 & 89,7 & 4 & 10,2 \\
Qualquer alimento diet e adoçante podem ser consumidos pelos & 29 & 74,3 & 10 & 25,6 \\
diabéticos à vontade & 23 & 58,9 & 16 & 41,0 \\
O diabético pode comer verduras e leguminosas livremente & 37 & 94,8 & 2 & 5,1 \\
O diabético pode comer farinha de mandioca livremente & 37 & 94,8 & 2 & 5,1 \\
O diabético pode comer macaxeira livremente & & & & \\
\hline
\end{tabular}


diabético. Portanto, $61,5 \%(\mathrm{n}=24)$ dos idosos acompanhados no Centro de Convivência de Idosos não eram acometidos por essa patologia.

Analisando o conhecimento alimentar dos indivíduos, observou-se que a maioria possui conhecimentos adequados sobre os tipos de alimentos que podem influenciar no tratamento do diabetes mellitus, como mostra a Tabela I.

Entre os avaliados, $51,2 \%(\mathrm{n}=20)$ disseram conhecer algum alimento/preparação que pode reduzir os riscos de acometimento ou auxiliar no tratamento do diabetes. No grupo dos indivíduos com diabetes, esse percentual mostrou-se ainda maior $(64,2 \% ; n=9)$.

Dentre os alimentos informados, o mais citado foi a berinjela, lembrada por $35 \%(\mathrm{n}=7)$ dos vinte participantes que afirmaram tal conhecimento. Os entrevistados citaram, ainda, alimentos como: soja, chá de carqueja, maracujá, feijão de corda, cebola, banana verde, quiabo, alface, laranja, semente de laranja comum torrada, água de banana pão e suco de limão com alho e cebola.

Dos vinte participantes que afirmaram ter o conhecimento de algum alimento/preparação que pode reduzir os riscos de acometimento ou auxiliar no tratamento do diabetes, $35 \%(n=7)$ afirmaram ter adquirido esse conhecimento por meio de televisão e conversa com conhecidos, mas citaram também o médico $(15 \% ; n=3)$, o programa de rádio $(5 \% ; \mathrm{n}=1)$, livros $(5 \% ; \mathrm{n}=1)$ e unidades de saúde $(5 \% ; n=1)$ como fontes de informação. A fonte de conhecimento mais citada pelos diabéticos foi a conversa com conhecidos $(33,3 \% ; n=3)$. Dos que citaram a fonte "conversa com conhecidos", $66,6 \%(\mathrm{n}=2)$ afirmaram ter feito uso do alimento indicado, mesmo sem ter certeza da confirmação científica quanto à finalidade terapêutica do alimento/preparação em questão. Além disso, $60 \%(\mathrm{n}=12)$ afirmaram já ter feito uso de algum desses, na expectativa de obter redução ou manter os níveis de glicose sanguínea.

Ainda assim, foi possível detectar que a maioria dos entrevistados $(56,4 \% ; n=22)$, diabéticos ou não, nunca esteve em uma consulta com um profissional de saúde para receber orientação sobre alimentação saudável e diabetes, e $58,9 \%(\mathrm{n}=23)$ nunca foram acompanhados por um nutricionista. Já entre os diabéticos, verificou-se que a maioria deles $(71,4 \% ; n=10)$ buscou orientação sobre alimentação e diabetes com algum profissional de saúde, porém, no que diz respeito ao acompanhamento nutricional, apenas $42,8 \%(n=6)$ afirmaram já terem sido acompanhados por um nutricionista.

Verificando o consumo alimentar de todos os entrevistados, constatou-se o maior consumo de alimentos de alto índice glicêmico do que de baixo índice glicêmico, como mostra a Tabela II.

Tabela II - Tipos de alimentos mais consumidos por frequentadores de um Centro de Convivência de Idosos na cidade de Sairé. Pernambuco, 2014.

\begin{tabular}{lcc}
\hline Alimentos & \multicolumn{2}{c}{ Consumo pela população } \\
\hline Alimentos de alto índice glicêmico & $\mathbf{n}$ & $\mathbf{\%}$ \\
Macaxeira & 31 & 79,4 \\
Melancia & 31 & 79,4 \\
Melão & 30 & 76,9 \\
Pão francês & 27 & 69,2 \\
Bolacha água e sal & 25 & 64,1 \\
Alimentos de baixo índice glicêmico & & \\
Feijão & 39 & 100 \\
Banana & 38 & 97,4 \\
Laranja & 34 & 87,1 \\
Leite integral & 21 & 53,8 \\
\hline
\end{tabular}

A farofa e a torrada são os alimentos de alto índice glicêmico menos ingeridos pela população avaliada, pois $89,7 \%(\mathrm{n}=35)$ e $58,9 \%(\mathrm{n}=23)$ dos indivíduos referiram não ingeri-los, respectivamente. Entre os alimentos de baixo índice glicêmico, o arroz integral, o leite desnatado, o iogurte, o iogurte diet e o refrigerante diet são consumidos por menos da metade dos participantes deste estudo, como mostra a Tabela III. 
Tabela III - Tipos de alimentos menos consumidos por frequentadores de um Centro de Convivência de Idosos na cidade de Sairé. Pernambuco, 2014.

\begin{tabular}{lcc}
\hline \multirow{2}{*}{ Alimentos } & \multicolumn{2}{c}{ Não consomem } \\
\cline { 2 - 3 } & $\mathbf{n}$ & $\mathbf{\%}$ \\
\hline Alimentos de alto índice glicêmico & 35 & 89,7 \\
Farofa & 23 & 58,9 \\
Torrada & & \\
Alimentos de baixo índice glicêmico & 30 & 76,9 \\
Arroz integral & 34 & 87,1 \\
Iorgute diet & 29 & 74,3 \\
Refrigerante diet & 26 & 66,6 \\
Iogurte & 25 & 64,1 \\
Leite desnatado & \multicolumn{2}{l}{} \\
\hline
\end{tabular}

Quanto à frequência de consumo semanal desses alimentos, viu-se que a macaxeira, a melancia e o melão são consumidos menos de 3 vezes na semana, representados por $83,8 \%(n=26), 61,2 \%(n=19)$ e $63,3 \%(n=19)$ dos indivíduos, respectivamente. No tocante ao feijão, grande parte $(69,2 \% ; n=27)$ consome-o diariamente. A banana é consumida por $42,1 \%(\mathrm{n}=16)$, de 3 a 5 vezes por semana.

No grupo dos diabéticos, assim como na população em geral, foi observado o consumo de mais alimentos de alto índice glicêmico do que de baixo índice glicêmico.
Porém, em relação à frequência de consumo, os alimentos de alto índice glicêmico são consumidos menos vezes e os de baixo índice glicêmico mais vezes por semana quando comparados com os indivíduos não diabéticos, como pode ser visto na Tabela IV.

Após análise de variância para avaliar os valores encontrados para ingestão de alimentos com baixo e alto índice glicêmico, não houve diferença estatisticamente significativa.

Tabela IV - Frequência de consumo alimentar semanal entre diabéticos e não diabéticos frequentadores de um Centro de Convivência de Idosos na cidade de Sairé. Pernambuco, 2014.

\begin{tabular}{lcccc}
\hline \multirow{2}{*}{ llimentos de alto índice glicêmico } & \multicolumn{2}{c}{ Diabéticos } & \multicolumn{2}{c}{ Não diabéticos } \\
\cline { 2 - 5 } Macaxeira & Vezes por semana & n (\%) & Vezes por semana & n (\%) \\
Melancia & $<3$ & $11(100)$ & $<3$ & $15(78,9)$ \\
Melão & $<3$ & $10(90,9)$ & 3 a 5 & $10(52,6)$ \\
Pão francês & $<3$ & $9(75)$ & $<3$ & $9(52,9)$ \\
Bolacha água e sal & 2 a 3 & $5(60)$ & Diariamente & $6(42,2)$ \\
Alimentos de baixo índice glicêmico & $<3$ & $4(44,4)$ & 3 a 5 & $7(46,6)$ \\
Feijão & & & & $14(58,3)$ \\
Banana & Diariamente & $12(85,7)$ & Diariamente & $12(52,1)$ \\
Laranja & $<3$ & $7(50)$ & 3 a 5 & $12(47,6)$ \\
Leite integral & 3 a 5 & $7(58,3)$ & $<3$ & $10(40)$ \\
\hline
\end{tabular}

\section{DISCUSSÃO}

Os indivíduos que frequentam o CCI, em sua maioria (74,3\%), não chegaram a concluir o ensino fundamental, caracterizando-se como uma população com baixo nível de escolaridade. Torna-se importante destacar que já foi observado na literatura a existência de associação entre a escolaridade e o conhecimento proporcionando melhores escolhas alimentares ${ }^{(11)}$. Entretanto, mesmo com a baixa escolaridade encontrada, o nível de conhecimento 
relacionado à alimentação e diabetes mellitus, de forma geral, foi considerado satisfatório, uma vez que a maioria das pessoas acertou as questões propostas referentes a esse tema. Em pesquisa na qual se analisaram os conhecimentos de usuários acometidos pelo diabetes mellitus na atenção primária a saúde, constatou-se que eles apresentam conhecimentos satisfatórios sobre fisiopatologia, práticas de autocuidado e complicações da doença, mas nem sempre isso é o suficiente para aderirem ao tratamento, pois relatam haver aspectos que interferem nesse processo ${ }^{(5)}$.

Com relação à renda, o maior número de indivíduos possui entre um e dois salários mínimos. Em estudo realizado com o intuito de caracterizar o consumo alimentar mais frequente na população brasileira, foi visto que há diferença no consumo alimentar entre indivíduos de maior e menor extrato de renda ${ }^{(12)}$. Em um estudo transversal de base populacional com 2.066 idosos de baixa renda do município de São Paulo, concluiu-se que o baixo poder aquisitivo foi associado à variedade limitada do consumo de frutas e hortaliças, mesmo sendo a amostra um grupo populacional relativamente homogêneo ${ }^{(13)}$.

Esse fator pode explicar a baixa ingestão dos alimentos diet, desnatados e integrais identificados no grupo do Centro de Convivência do Idoso, uma vez que são alimentos de maior custo se comparados aos demais questionados. Uma pesquisa nacional sobre o uso do orçamento familiar entre 2008 e 2009 também observou que o maior consumo de frutas, verduras, leite e derivados desnatados aumentava muito com a renda ${ }^{(14)}$.

De maneira geral, uma boa parcela afirmou o conhecimento de algum alimento/preparação que reduza os riscos de desenvolvimento ou interfira positivamente no diabetes. Esse conhecimento, em sua maior parte, vem sendo adquirido por meio da televisão. Um estudo observacional e descritivo mostrou, ao avaliar propagandas alimentícias de duas emissoras de televisão do canal aberto brasileiro, que, através de mensagens persuasivas, atraentes e marcantes, a mídia televisiva possui influência mais duradoura que a de familiares nas escolhas alimentares, e esta não tem contribuído para um estilo de vida saudável entre a população brasileira ${ }^{(15)}$.

Quando avaliados apenas os indivíduos diabéticos, o conhecimento sobre alimentos foi ainda maior, o que pode ser justificado pelo fato de estes serem acometidos pela patologia. E o principal meio de difusão da informação foi através de conversas informais com conhecidos. $\mathrm{O}$ convívio social leva a um conjunto de crenças, entre elas, as primitivas, que são aquelas aprendidas pelo encontro direto com o objeto da crença e reforçadas por consenso social, representando as verdades básicas do indivíduo. Tais crenças foram identificadas entre pacientes diabéticos como indicadores de baixa adesão à terapia nutricional ${ }^{(16)}$.
No presente estudo, identificou-se que a maior parte dos que fazem uso do alimento em que possui conhecimento são as pessoas já acometidas pelo diabetes mellitus $(88,8 \%)$, a fim de obterem a cura ou melhora do quadro clínico, comparando-os com a parcela total que respondeu positivamente a essa questão. Os dados corroboram com o estudo realizado em Teresina-PI, que analisou as dificuldades encontradas para mudança de práticas alimentares entre diabéticos atendidos em um consultório de nutrição do Sistema Único de Saúde no município de Teresina. Observou-se que a tradição está fortemente ligada à forma como os indivíduos lidam com o diabetes, uma vez que todos os avaliados fizeram referência à prática da medicina popular, a qual foi observada forte credibilidade e uso mais ou menos indiscriminado ${ }^{(17)}$.

Por outro lado, um estudo realizado no ano de 2013 mostrou que $65 \%$ dos indivíduos diabéticos hospitalizados em um Hospital das Clínicas que faziam uso de alimentos funcionais para o controle do diabetes não receberam nenhuma indicação para fazê-lo, e apenas $9 \%$ disseram que a indicação foi realizada por conhecidos ${ }^{(18)}$.

$\mathrm{O}$ alimento mais lembrado pelo grupo foi a berinjela. Em uma revisão da literatura, na qual estudaram e analisaram artigos publicados em bases de dados, livros e revistas científicas, e procuraram justificar as características da berinjela como um alimento funcional, foi visto que muitos estudos revelam que esse alimento possui em sua composição química componentes que o tornam importante na prevenção e no tratamento de algumas doenças crônicas não transmissíveis, como o diabetes ${ }^{(19)}$.

Entre outros alimentos, a soja, a cebola e o suco de limão com cebola e alho estiveram presentes. Em pesquisa enfatizando as propriedades dos alimentos funcionais e seu papel na prevenção e controle da hipertensão e diabetes, dados afirmam que as fibras da soja regulam os níveis de glicose no sangue ${ }^{(20)}$, enquanto a cebola e o alho são considerados alimentos funcionais importantes no controle do diabetes ${ }^{(18)}$. Dessa forma, pudemos verificar que esse é um conhecimento ainda vago no grupo. Por isso, ressaltase a importância da realização de atividades educativas que possam esclarecer e ampliar os conhecimentos dos indivíduos sobre alimentos funcionais, com as devidas comprovações científicas, e a forma como esses conhecimentos devem ser utilizados para que possam realmente auxiliar na prevenção e/ou tratamento do diabetes mellitus.

A busca pelo profissional de saúde para receber orientações sobre alimentação e diabetes é mais comum entre os diabéticos $(71,4 \%)$, enquanto que a maior parte da população geral nunca teve contato para essa finalidade. Tendência vista em estudo no qual avaliaram a população residente na Vila Gaúcha, em Porto Alegre, e identificaram 37 indivíduos com diabetes mellitus diagnosticados pelo 
médico, dentre os quais $81,1 \%$ receberam orientação de algum profissional sobre a dieta e $64,9 \%$ afirmaram ter se consultado com o médico por conta da doença no ano anterior à realização da pesquisa ${ }^{(21)}$.

No que diz respeito ao acompanhamento nutricional, a maior parte dos indivíduos referiu nunca ter feito $(58,9 \%)$. Já entre os diabéticos, foi visto que, apesar de $42,8 \%$ afirmarem já ter feito acompanhamento com o nutricionista, este ainda é considerado um dado insatisfatório, pois a doença necessita que o indivíduo mantenha uma alimentação adequada e equilibrada para que seja possível mantê-la controlada, com o objetivo de evitar ou retardar suas complicações.

Evidências indicam que o índice glicêmico dos alimentos, se associado ao planejamento dietético de pacientes diabéticos, ajuda a melhorar o controle glicêmico no indivíduo e, se mantido permanentemente, pode prevenir e/ou retardar o surgimento de complicações decorrentes do diabetes $^{(22)}$. Com relação ao consumo alimentar entre os indivíduos do Centro de Convivência, há uma tendência ao consumo de mais alimentos com alto índice glicêmico do que de baixo índice glicêmico. Contudo, sabe-se que alimentos de alto índice glicêmico possuem maior efeito sobre a glicemia, por serem digeridos e absorvidos rapidamente, e que há fatores intrínsecos e extrínsecos ao alimento que podem influenciar nesse índice ${ }^{(23)}$.

A macaxeira e a melancia, que fazem parte do grupo de alimentos de alto índice glicêmico, são consumidas pela maioria dos entrevistados, porém, com frequência de consumo considerada baixa (menos de três vezes na semana). Já o pão francês e a bolacha apresentam elevada frequência de consumo, com uso diariamente e de 3 a 5 vezes por semana, respectivamente.

No grupo dos alimentos de baixo índice glicêmico, destacamos que o feijão é consumido pela maioria dos entrevistados diariamente $\mathrm{e}$, independentemente da frequência, todos fazem uso desse alimento, pois ele faz parte da cultura alimentar do brasileiro. Uma análise estratificada realizada por região analisou dados de indivíduos selecionados para participar do Inquérito Nacional de Alimentação (INA) e demonstrou que o feijão e o pão de sal estão entre os cinco alimentos com maior prevalência de consumo no Brasil ${ }^{(12)}$. Identificamos que a banana e a laranja também são muito consumidas pelo grupo $-97,4 \%$ e $87,1 \%$, respectivamente. Estudo mostra que, entre os vinte alimentos mais prevalentes no consumo da população brasileira, a banana é a principal fruta em todas as faixas de idade, e somente os idosos citaram uma segunda fruta nesse grupo: a laranja ${ }^{(12)}$.

A ingestão de leite integral mostrou-se por boa parte dos indivíduos $(53,8 \%)$, todavia, com baixa frequência semanal (menos de três vezes). Uma pesquisa realizada em todas as capitais dos estados brasileiros e no Distrito Federal identificou que a frequência de consumo de leite com teor integral de gordura pela população adulta $(\geq 18$ anos) foi elevada em todas as cidades estudadas $(53,8 \%)$, sendo maior entre homens que entre mulheres, e que o consumo tendeu a diminuir com o aumento da idade ${ }^{(24)}$.

Quando são observados os participantes diabéticos, verifica-se que os alimentos de alto índice glicêmico são consumidos menos vezes por semana que os alimentos de baixo índice glicêmico em comparação com os que não são diabéticos. Tendência vista em Bauru-SP, onde indivíduos portadores de diabetes relatam consumir mais frutas e vegetais, laticínios desnatados, refrigerantes dietéticos, oleaginosas e azeite, e menos doces e refrigerantes açucarados, quando comparados aos indivíduos não diabéticos ${ }^{(25)}$. Todavia, em estudo também no interior do estado de São Paulo, que avaliou o consumo alimentar de indivíduos com diabetes tipo 2, verificou-se que a maioria consome alimentos com alto teor de carboidrato, como batata, mandioca, farinha branca e arroz polido ${ }^{(26)}$. Outro trabalho realizado num hospital universitário do Rio de Janeiro mostrou que dietas de baixo índice glicêmico são capazes de melhorar o controle glicêmico em pacientes com diabetes tipo $1^{(27)}$.

Este estudo apresentou como possível limitação o aspecto fisiológico do envelhecimento, em que a perda de memória se faz presente e poderia influenciar nas respostas sobre o consumo alimentar dos idosos.

Tendo em vista a progressão importante da mortalidade por diabetes no Brasil, afirma-se a necessidade de que esses assuntos estejam no centro das políticas públicas e sugerese, assim, a intensificação das ações de promoção da saúde no grupo, pois a persistência e a continuidade desses atos podem ocasionar mudanças nos hábitos de vida e contribuir para prevenção do diabetes.

\section{CONCLUSÃO}

O nível de conhecimento em relação à alimentação e ao diabetes mellitus dos idosos foi considerado adequado, mas observou-se que fatores socioeconômicos e culturais podem interferir na adesão à terapia nutricional do diabetes ou, ainda, nas escolhas alimentares dos indivíduos. Contudo, o consumo alimentar se mostra mais adequado entre os indivíduos com diabetes, uma vez que estes apresentam uma menor frequência de consumo de alimentos do tipo de alto índice glicêmico e maior frequência de consumo de alimentos de baixo índice glicêmico, quando comparados à população não diabética. 


\section{REFERÊNCIAS}

1. Silva SMCS, Mura JDP. Tratado de Alimentação Nutrição e Dietoterapia. 2a ed. São Paulo: Roca; 2010.

2. Sociedade Brasileira de Diabetes - SBD. Diretrizes da Sociedade Brasileira de Diabetes 2009. $3^{\mathrm{a}}$ ed. Itapevi: A. Araújo Silva Farmacêutica; 2009.

3. Ministério da Saúde (BR), Secretaria de Atenção à Saúde, Departamento de Atenção Básica Diabete Mellitus. Brasília: Ministério da Saúde; 2006.

4. Cotta RMM, Reis RS, Batista KCS, Dias G, Alfenas RCG, Castro FAF. Hábitos e práticas alimentares de hipertensos e diabéticos: repensando o cuidado a partir da atenção primária. Rev Nutr. 2009;22(6):823-35.

5. Pereira PF, Cordeiro PMC, Souza LFD, Torres HC. Conhecimentos dos usuários com diabetes mellitus na atenção primária à saúde. Convibra Saúde 2012 [acesso em 2014 Mar 04]; Disponível em: http://www.convibra. org/saude_2012.asp?ev $=77 \&$ lang $=$ pt\&ano $=2012$

6. Borges TT, Rombaldi AJ, Knuth AG, Hallal PC. Conhecimento sobre fatores de risco para doenças crônicas: estudo de base populacional. Cad Saúde Pública. 2009;25(7):1511-20.

7. Anunciação PC, Braga PG, Almeida PS, Lobo LN, Pessoa MC. Avaliação do conhecimento sobre alimentação antes e após intervenção nutricional entre diabéticos tipo 2. Rev Baiana Saúde Pública. 2012;36(4):986-1001.

8. Foster-Powell K, Holt SHA, Brand Miller JC. International table of glycemic index and glycemic load values: 2002. Am J Clin Nutr. 2002;76(1):5-56.

9. Brand Miller JC, Burani J, Foster J, Powell K, Holt SHA. The new glucose revolution: complete guide to glycmic index values. $3^{\text {th }}$ ed. New York: Marlowe \& Company; 2003.

10. Dias VM, Pandini JA, Nunes RR, Sperandei SLM, Portella ES, Cobas RA, Gomes MB. Influência do índice glicêmico da dieta sobre parâmetros antropométricos e bioquímicos em pacientes com diabetes tipo 1. Arq Bras Endocrinol Metab. 2010;54(9):801-6.

11. Kümpel DA, Silveira MM, Rocha JP, Scariot M, Portella MR, Pasqualotti A. Perfil alimentar de idosos frequentadores de um grupo de terceira idade. Rev Contexto \& Saúde. 2011;10(20):361-6.

12. Souza AM, Pereira RA, Yokoo EM, Levy RB, Sichieri R. Alimentos mais consumidos no Brasil: Inquérito Nacional de Alimentação 2008-2009. Rev Saúde Pública. 2013;47(Supl 1):190S-19S.
13. Viebig RF, Pastor-Valero M, Scazufca M, Menezes PR. Consumo de frutas e hortaliças por idosos de baixa renda na cidade de São Paulo. Rev Saúde Pública. 2009;43(5):806-13.

14. Instituto Brasileiro de Geografia e Estatística - IBGE Pesquisa de orçamentos familiares 2008-2009: análise do consumo alimentar pessoal no Brasil. Rio de Janeiro: IBGE; 2011.

15. Santos CC, Stuchi RAG, Arreguy-Sena C, Pinto NAVD. A influência da televisão nos hábitos, costumes e comportamento alimentar. Cogitare Enferm. 2012;17(1):65-71.

16. Pontieri FM, Bachion MM. Crenças de pacientes diabéticos acerca da terapia nutricional e sua influência na adesão ao tratamento. Ciênc Saúde Coletiva. 2010;15(1):151-60.

17. Santos AFL, Araújo JWG. Prática alimentar e diabetes: desafios para a vigilância em saúde. Epidemiol Serv Saúde. 2011;20(2):255-63.

18. Zaparolli MR, Nascimento NC, Baptista DR, Vayego SA. Alimentos funcionais no manejo da diabetes mellitus. Rev Ciênc Saúde. 2013;6(1):12-7.

19. Carvalho MMS, Lino LLA. Avaliação dos fatores que caracterizam a berinjela (Solanum melongena L.) como um alimento funcional. Nutrire Rev Soc Bras Aliment Nutr. 2014;39(1):130-43.

20. Basho SM, Bin MC. Propriedades dos alimentos funcionais e seu papel na prevenção e controle da hipertensão e diabetes. Interbio. 2010;4(1):48-58.

21. Mielczarski RG, Costa JSD, Olinto MTA. Epidemiologia e organização de serviços de saúde: diabetes mellitus numa comunidade de Porto Alegre. Ciênc Saúde Coletiva. 2012;17(1):71-8.

22. Silva FM, Steemburgo T, Azevedo MJ, Mello VD. De. Papel do índice glicêmico e da carga glicêmica na prevenção e no controle metabólico de pacientes com diabetes mellitus tipo 2. Arq Bras Endocrinol Metab. 2009;53(5):560-71.

23. Nascimento VB. Emprego do índice glicêmico e carga glicêmica dos alimentos: uma alternativa nas dietas de pacientes com doenças crônicas? Rev Assoc Bras Nutr. 2012;4(5):48-53.

24. Ministério da Saúde (BR), Secretaria de Vigilância em Saúde, Departamento de vigilância de doenças e agravos não transmissíveis e Promoção de Saúde. Vigitel Brasil 2012: Vigilância de fatores de risco e proteção para doenças crônicas por inquérito telefônico. Brasília: Ministério da Saúde; 2013. 
25. Nishimura RY, Damião R, Gimeno SGA, Ferreira SRG, Sartorelli DS. Grupos de alimentos para investigação de risco para diabetes tipo 2 e doenças associadas. Rev Bras Epidemiol. 2011;14(3):531-6.

26. Barbieri AFS, Chagas IA, Santos MA, Teixeira CRS, Zanetti ML. Consumo alimentar de pessoas com diabetes mellitus tipo 2. Rev Enferm. 2012;20(2):15560 .

27. Dias VM, Pandini JA, Nunes RR, Sperandei SLM, Portella ES, Cobas RA, et al. Influência do índice glicêmico da dieta sobre parâmetros antropométricos e bioquímicos em pacientes com diabetes tipo 1. Arq Bras Endocrinol Metab. 2010; 54(9):801-6.
Endereço do primeiro autor:

Maria Andressa Gomes Barbosa

Centro Universitário do Vale do Ipojuca - UNIFAVIP/

DeVry

Av. Adjar da Silva Casé, 800

Bairro: Indianópolis

CEP: 55.024-740 - Caruaru - PE - Brasil

E-mail: andressa.gomes2009@hotmail.com

Endereço para correspondência:

Georgia Karoline Cavalcante Galvão

Centro Universitário do Vale do Ipojuca- UNIFAVIP/

DeVry

Av. Adjar da Silva Casé, 800

Bairro: Indianópolis

CEP: 55.024-740 - Caruaru - PE - Brasil

E-mail: ggalvao@unifavip.edu.br 